\title{
LA QUESTIONE DIMENSIONALE PER LE IMPRESE ITALIANE \\ NELLO SCENARIO COMPETITIVO GLOBALE
}

di Fabio Musso

\section{La dimensione d'impresa nel sistema manifatturiero italiano}

Il ricco dibattito che si è sviluppato in questi anni attorno al ruolo che il sistema industriale italiano può ricoprire nell'economia globalizzata ha messo in evidenza, fra i numerosi aspetti emersi, la vulnerabilità della piccola dimensione d'impresa. Diversi studi hanno rilevato le capacità delle medie imprese di affermarsi efficacemente nel mercato nazionale e in quelli esteri (Ferrucci e Guercini, 2013; Fortis e Quadrio Curzio, 2006; Pucci, Simoni e Zanni, 2013; Resciniti e Matarazzo, 2012), così come i dati, anche recenti, che testimoniano la competitività internazionale del sistema manifatturiero italiano ${ }^{1}$, traggono alimento per lo più dai risultati delle medie imprese. Le piccole imprese, che rappresentano di gran lunga la quota più rilevante della struttura industriale italiana ${ }^{2}$, stanno invece incontrando crescenti difficoltà. La misura di questa difficoltà è nel processo di selezione in corso, che nell'ultimo decennio ha portato alla scomparsa di oltre un quarto delle micro imprese manifatturiere, contro un andamento del numero di medie e grandi imprese che, se pure anch'esso in calo, si è fermato a una riduzione di meno del $20 \%$ (tab. 1).

Tab. 1 - Numero di imprese per classi dimensionali. Confronto 2001-2011

\begin{tabular}{lrrrr}
\hline & 2001 & 2011 & Diff. & Diff. \% \\
\hline Micro imprese (meno di 10 addetti) & 447.859 & 334.914 & -112.945 & $-25,2 \%$ \\
Piccole imprese (10-49 addetti) & 82.628 & 63.968 & -18.660 & $-22,6 \%$ \\
Medie imprese (50-49 addetti) & 10.872 & 8.778 & -2.094 & $-19,3 \%$ \\
Grandi imprese (oltre 250 addetti) & 1517 & 1.227 & -290 & $-19,1 \%$ \\
\hline
\end{tabular}

Fonte: Istat, Censimento dell'Industria e dei servizi, anni 2001 e 2011

1 Si vedano, a tale proposito, i numerosi rapporti delle Fondazione Edison.

2 Le imprese manifatturiere con meno di 50 addetti sono in Italia il 97,6\% del totale. Fonte: Istat, Censimento dell'Industria e dei servizi $2011<$ http://www.istat.it>.

ECONOMIA E SOCIETÀ REGIONALE - XXXII (3) 2014 - DIBATTITO SU “LE SFIDE DELL'INTERNALIZZAZIONE NEL NUOVO MILLENNIO" 
Di fronte a questo scenario, occorre prendere atto che la piccola dimensione di impresa non è più un modello vincente come lo era stato in passato, e che senza dubbio vanno favoriti e per quanto possibile replicati modelli riconducibili alla media e alla grande impresa. Resta però la questione delle imprese esistenti, che non possono essere lasciate di fronte al processo di drastica selezione in atto senza tentare di dotarle di adeguati strumenti di rilancio. Esse, d'altra parte, difficilmente potranno evolvere, se non in piccola parte, verso un maggiore livello dimensionale.

Nel pensare a percorsi che abbiano effetti nel breve-medio termine e che, allo stesso tempo, possano stimolare una graduale crescita delle imprese, ci sono tre temi che meritano di essere ripesi. Si tratta di temi ampiamente dibattuti ma che conservano la loro attualità in una fase, come quella attuale, in cui buona parte del futuro economico del Paese appare legata alla capacità competitiva che il sistema manifatturiero nazionale, cioè le imprese, saprà esprimere nello scenario competitivo globale.

Il primo tema riguarda le forme di aggregazione che possono essere realizzate per superare i limiti della piccola dimensione e allo stesso tempo salvaguardare l'autonomia imprenditoriale: consorzi, contratti i rete e altre forme di alleanza strategica per fare massa critica su fronti che richiedono investimenti e adeguate competenze e strutture organizzative.

Il secondo tema è quello del sostegno pubblico all'internazionalizzazione, che è in fase di profondo ripensamento in funzione di un'auspicabile razionalizzazione, di cui può essere l'occasione per rivedere $\mathrm{i}$ criteri di base.

Il terzo tema è quello dei territori, che dal concetto di distretto industriale vanno riletti come sistemi, o ecosistemi, in grado di alimentare processi di valorizzazione della conoscenza secondo regole di interazione che stanno cambiando, soprattutto in seguito ai cambiamenti delle tecnologie di comunicazione.

\section{Consorzi, reti e alleanze per la valorizzazione del made in Italy}

Le diverse forme di alleanza fra piccole e medie imprese per lo sviluppo dei mercati esteri, di cui i consorzi rappresentano l'espressione più significativa, sono state ampiamente analizzate in passato, ma recentemente si avverte l'esigenza di verificarne il ruolo e l'efficacia alla luce delle dinamiche della competizione internazionale (Guercini e Woodside, 2012). Infatti, l'intensificarsi e il complessificarsi della concorrenza in tutti i mercati mondiali richiedono alle imprese un maggiore sforzo in termini di risorse e competenze per competere adeguatamente. 
Questo vale a maggior ragione per i mercati emergenti, dove occorre adottare strategie commerciali articolate e si devono fronteggiare difficoltà legate alla maggiore distanza geografica, culturale, normativa e nelle infrastrutture di marketing, oltre che psicologica, che li caratterizza. Simili fattori si rivelano critici soprattutto per le imprese minori, che fanno fatica a disporre di risorse - finanziarie, di competenze, organizzative - utili al presidio di mercati per i quali strategie di ingresso indirette si rivelano sempre meno adeguate.

Alla luce di tutto questo, la funzione dei consorzi per lo sviluppo dei mercati esteri tende a rivalutarsi: se in passato le aggregazioni fra imprese potevano rappresentare un'opportunità per rafforzare il vantaggio competitivo, oggi diventano una necessità, e da più parti viene richiamata l'esigenza di adottare modalità collaborative nuove sia nell'ambito di reti e relazioni informali, sia in quello delle collaborazioni formalizzate con e senza forma giuridica: consorzi, associazioni temporanee di imprese, jointventures, contratti di rete, accordi.

In realtà, pur essendoci consapevolezza da parte di imprese e istituzioni circa la rinnovata importanza che l'azione consortile può rivestire, un cambiamento nei modelli di aggregazione per lo sviluppo dei mercati esteri ha ancora difficoltà a manifestarsi (Musso, Francioni e Pagano, 2012). Uno dei principali freni continua a essere la diffidenza da parte delle imprese per timore di perdere autonomia, accompagnata da dinamiche concorrenziali e non di rado conflittuali che prevalgono su quelle collaborative.

Gli stessi organismi aggregativi manifestano dei limiti, fra cui la prevalenza di finalità promozionali rispetto a obiettivi di sviluppo stabile del mercato, e la difficoltà a realizzare programmi collegando entro un chiaro disegno strategico analisi di mercato, obiettivi, scelte di posizionamento e strumenti operativi. Un altro aspetto che emerge riguarda il rapporto spesso capovolto fra obiettivi e condizioni di finanziamento per perseguirli, nel senso che i finanziamenti disponibili finiscono per diventare il principale riferimento rispetto a cui intraprendere azioni non necessariamente esaustive né coerenti.

Tenendo conto del perdurare di queste criticità, occorre un percorso di cambiamento innanzitutto all'interno delle imprese, a partire dalla percezione che esse hanno della condivisione di progetti, ma anche all'esterno, negli assetti degli organismi aggregativi.

Il primo punto rimanda al carattere degli imprenditori e alla cultura che li accompagna, per i quali stimoli formativi e informativi diffusi e costanti possono rappresentare il principale strumento da utilizzare. Con riferimento al secondo punto, il passaggio da compiere è da organismi di promozio- 
ne dell'export ad alleanze per l'internazionalizzazione, combinando in modo sinergico le attività promozionali con iniziative in grado di assicurare continuità nei rapporti di mercato. A tale fine si rivelano variabili critiche la disponibilità di adeguate risorse organizzative dell'organismo di coordinamento, l'esistenza di una rete di rapporti con interlocutori sia economici che istituzionali, operanti a livello nazionale e direttamente nei mercati obiettivo e, soprattutto, una logica di fondo caratterizzata da obiettivi commerciali quale punto di riferimento prioritario, in funzione del quale cercare finanziamenti e sfruttare anche eventuali benefici fiscali, ma non viceversa.

\section{Verso un nuovo modello di sostegno pubblico per lo sviluppo dei mercati esteri}

In un momento in cui due pilastri del sostegno pubblico all'internazionalizzazione, l'Ice e le Camere di Commercio, sono oggetto di un profondo ripensamento, vale forse la pena riflettere sulla validità del modello finora seguito per sostenere le imprese nelle attività di promozione e sviluppo di nuovi mercati, in funzione di futuri assetti che auspicabilmente possano meglio rispondere alle esigenze del mondo produttivo.

Il modello finora seguito può essere inquadrato su due livelli: un livello centrale, con Ministeri ed enti dedicati (Ice, Simest, Sace, Enit...), e un livello locale che ha visto coinvolte le Camere di Commercio e in buona misura le amministrazioni locali, soprattutto le Regioni, ma non di rado anche le Provincie o singoli Comuni.

Oltre al problema ampiamente noto della frammentazione dei soggetti coinvolti e del loro scarso coordinamento, soprattutto a livello locale, ce n'è un altro, meno evidente ma altrettanto critico. Si tratta del tipo di supporto che viene offerto alle imprese. Per entrambi i livelli di intervento i principali strumenti adottati sono l'organizzazione di partecipazioni a fiere e la realizzazione di missioni istituzionali. Le prime sono più direttamente legate ai settori e ai relativi operatori, mentre le seconde permettono di entrare in rapporto con interlocutori privilegiati dei Paesi esteri, come organi di governo centrale o locale, i quali possono facilitare lo sviluppo di relazioni economiche e l'apertura di nuovi spazi di mercato.

Questa impostazione si rivela efficace per le imprese di grandi dimensioni che hanno competenze, risorse, capacità organizzativa e chiarezza strategica per riuscire a sfruttare l'apertura istituzionale e sviluppare rapporti su un terreno di più concreta operatività, riuscendo nel tempo a consolidarli. 
Lo stesso modello, applicato a livello locale, non è altrettanto efficace. Se è vero che localmente possono meglio essere individuati Paesi e settori maggiormente compatibili con le specializzazioni produttive prevalenti di un singolo territorio, di cui le associazioni di categoria si fanno portavoce partecipando ai progetti, ciò che non funziona è limitare il supporto al solo momento del contatto iniziale. Occorre ricordare che le iniziative territoriali sono rivolte per lo più alle imprese minori, quelle non coinvolte nei grandi progetti nazionali, e tali imprese hanno difficoltà a muoversi autonomamente in mercati nuovi. Sia che partecipino a una fiera, sia che vengano coinvolte in un viaggio/missione di rappresentanza, una volta incontrati i potenziali interlocutori manca loro la capacità di procedere con le proprie forze, con personale adeguato, capacità organizzativa, competenze di marketing e risorse per sviluppare e consolidare i rapporti.

Le grandi imprese sanno come fare e hanno i mezzi per farlo, le piccole spesso non hanno la consapevolezza né i mezzi.

Nel ripensare il sistema di supporto all'internazionalizzazione, quindi, occorrerebbe tenere maggiormente conto di questo dualismo, seguendo un modello che a livello centrale, e a favore delle grandi imprese - e comunque dell'indotto di piccole e medie imprese che esse alimentano -, favorisca soprattutto l'avvio di contatti e relazioni qualificate, mentre a livello decentrato indirizzi risorse verso strumenti più operativi. Questo, se realizzato con soluzioni tarate sulle caratteristiche dimensionali e settoriali delle imprese, potrebbe contribuire a ridurre la distanza fra aspettative e ciò che viene offerto, permettendo anche di farne riconoscere il valore.

Un segnale positivo in tal senso viene dal recente orientamento che il Ministero dello Sviluppo Economico, in collaborazione con Ice Agenzia, sta seguendo nel mettere a punto interventi concreti che diano alle imprese la possibilità di essere accompagnate nei percorsi di sviluppo all'estero. La direzione è quella giusta per fornire l'aiuto necessario, anche se poi si dovrà pure stimolare l'accrescimento delle competenze interne per favorire maggiore autonomia.

\section{Dai distretti agli ecosistemi di innovazione}

Il tema dei territori richiama il concetto di distretto industriale su cui molto si è dibattuto ma su cui molto c'è ancora da comprendere, soprattutto a causa dei profondi cambiamenti nei processi di comunicazione e logistici che stanno portando a continue ridefinizioni delle coordinate di riferimento entro cui collocare il fenomeno.

È indubbio che i contesti locali possono giocare un ruolo determinante nell'influenzare i percorsi di crescita dimensionali delle imprese. Se in 
passato l'appartenenza a un distretto poteva rappresentare una sorta di elemento compensatore della piccola dimensione aziendale, soprattutto sul piano delle economie industriali, favorendo quindi il mantenimento delle dimensioni ridotte, oggi molte cose sono cambiate. Molti dei vantaggi dei distretti sono venuti meno, soprattutto in seguito all'affermarsi delle catene del valore globali, e questo spinge a cercare nei sistemi economici locali elementi propulsivi a favore della crescita delle imprese.

I fronti a cui guardare sono quelli dell'innovazione, dell'efficienza dei processi di approvvigionamento e produttivi, e della capacità di sviluppare rapporti con i mercati e i relativi intermediari di riferimento. Ciò che può essere fatto su tali fronti è valorizzare i processi di diffusione e contaminazione delle conoscenze, a favore dei quali il concetto di polisettorialità dei sistemi locali assume un significato particolarmente rilevante. Infatti, laddove si rende possibile ridurre le separazioni settoriali, possono scaturire innovazioni in grado di combinare trasversalmente tradizioni artigianali e manifatturiere, da una parte, e tecnologie avanzate, dall'altra. Si pensi, per esempio, alle tecnologie per la domotica e l'illuminazione applicate al settore dell'arredamento, oppure all'utilizzo di nuovi materiali nel settore dell'abbigliamento o, sempre nel settore dell'abbigliamento, all'inserimento di dispositivi tecnologici in grado di rilevazioni geolocalizzate e monitoraggi sui parametri biologici.

Tali aree di innovazione vanno poi coniugate con l'Internet delle cose, che permette di collegare fra loro persone, processi, dati e oggetti tecnologici. Ne derivano anche in questo campo opportunità aggiuntive di innovazione sull'efficienza, la sicurezza, la velocità dei processi decisionali, l'erogazione di servizi.

Anche lo sviluppo e il presidio dei mercati vanno giocati secondo nuove regole, con processi di comunicazione e commerciali che sempre più risultano condizionati dai social network. L'affermarsi di modelli di comunicazione fra pari diventa allora un ulteriore riferimento a cui guardare per raggiungere i mercati e affermare le identità di marca e di origine secondo logiche diverse da quelle tradizionali.

La variabile chiave di tutti gli aspetti menzionati è il cambiamento, in funzione del quale gli assetti dei sistemi territoriali devono continuamente adattarsi. Per questa ragione il concetto di ecosistema di innovazione può richiamare il carattere evolutivo, adattivo e selettivo dei sistemi ecologici, secondo modelli in grado di analizzare gli agglomerati di imprese e il loro comportamento di co-evoluzione (Carroll e Hannan, 2000; Moore, 1996; 2006; Lewin, Long e Carroll, 1999; Basole, 2009; Iansiti e Levien, 2004; Tee e Gawer, 2009; Jacobides, Knudsen e Augier, 2006). 


\section{Considerazioni conclusive}

La questione dimensionale resta un aspetto fondamentale per valutare correttamente i processi di internazionalizzazione delle imprese italiane. Di fronte all'esigenza di superare gli ostacoli derivanti da una dimensione eccessivamente ridotta, oltre alla crescita per linee interne che deve essere in ogni caso favorita e stimolata, la via delle alleanze strategiche con altre imprese resta uno dei percorsi prioritari, e questo vale non solo per attività di sviluppo dei mercati esteri ma anche per l'innovazione, il miglioramento degli standard qualitativi, la logistica e tutti i casi in cui la dimensione di scala garantisce maggiore efficacia delle funzioni svolte.

Come si è visto, le tecnologie per la comunicazione e il modo in cui esse evolvono giocano un ruolo cruciale, e questo significa innanzitutto dover mettere le imprese in condizione di sfruttarne le potenzialità. Per l'Italia c'è un problema di infrastrutture che alimenta quello di un'ancòra bassa diffusione nell'uso di tecnologie evolute. Un problema culturale che si incrocia con quello infrastrutturale, per il quale occorre indubbiamente colmare dei ritardi.

Per quanto riguarda la cultura, inoltre, va senz'altro richiamata anche la necessità di fare evolvere le competenze manageriali all'interno delle imprese più piccole, che possono comunque trovare spazio. A tal fine il momento del ricambio generazionale costituisce un passaggio critico, così come il rapporto con il sistema della formazione superiore e soprattutto universitaria, verso cui le forme di collaborazione vanno senz'altro intensificate.

Un'ulteriore questione di rilievo riguarda il rapporto con il sistema finanziario, rispetto al quale le piccole imprese risultano particolarmente penalizzate. Migliori condizioni di accesso al credito e maggiore trasparenza nel rapporto fra imprese e intermediari finanziari sono essenziali non solo per sostenere strategie di crescita, ma anche per affrontare scelte di internazionalizzazione che richiedono investimenti consistenti.

Un simile quadro, per l'articolazione dei fattori che lo influenzano, richiede un lavoro che sia in grado di sintonizzare ciò che compete alle imprese, e che possono affrontare con le proprie risorse, con ciò che può essere fatto dall'esterno. I fronti strategici segnalati, con i relativi vincoli e criticità, richiedono necessariamente una complementarietà fra imprese, soggetti esterni e istituzioni, affinché le condizioni interne ed esterne alle imprese possano evolvere in funzione di obiettivi confluenti e fra loro coerenti.

\section{Riferimenti bibliografici}

Basole R.C. (2009). Visualization of Interfirm Relations in a Converging Mobile Ecosystem. Journal of Information Technology (24)2: 144-159, doi:10.1057/jit.2008.34. 
Carroll G.R. e Hannan M.T. (2000). The Demography of Corporations and Industries. Princeton (NJ): Princeton University Press.

Censis (2014). Quarantottesimo Rapporto sulla situazione sociale del Paese. Milano: FrancoAngeli.

Coltorti F. e Garofoli G. (2011). Le medie imprese in Europa. Economia Italiana, 1: $187-223$.

Ferrucci L. e Guercini S. (2013). Le medie imprese tra continuità strategica e cambiamento nella crisi economica mondiale. Mercati e competitività, 3: 11-24, doi: 10.3280/MC2013-003002.

Fortis M. e Quadrio Curzio A., a cura di (2006). Industria e distretti. Un paradigma di perdurante competitività italiana. Bologna: il Mulino.

Garofoli G. (2014). Economia e politica economica in Italia. Lo sviluppo economico italiano dal 1945 ad oggi. Milano: FrancoAngeli.

Guercini S. e Woodside A.G. (2012). A strategic supply chain approach: consortium marketing in the Italian leatherwear industry. Marketing Intelligence \& Planning, 30: 700-716, doi: 10.1108/02634501211273814.

Iansiti M. e Levien R. (2004). The Keystone Advantage: What the New Dynamics of Business Ecosystems Mean for Strategy, Innovation and Sustainability. Boston (MA): Harvard Business School Press.

Jacobides M., Knudsen T. e Augier M. (2006). Benefiting from Innovation: Value Creation, Value Appropriation and the Role of Industry Architectures. Research Policy (35)8: 1200-1221, doi:10.1016/j.respol.2006.09.005.

Lewin A.Y., Long C.P. e Carroll T.N. (1999). The coevolution of new organizational forms. Organization Science, 10(5): 535-550, doi: 10.1287/orsc.10. 5.535 .

Moore J.F. (1996). The Death of Competition: Leadership and Strategy in the Age of Business Ecosystems. New York: HarperCollins.

Moore J.F. (2006). Business ecosystems and the view from the firm. The Antitrust Bulletin, 51(1): 31-75.

Musso F., Francioni B. e Pagano A. (2012). The Role Of Country-Of-Origin in Supporting Export Consortia in Emerging Markets. In: Bertoli G. e Resciniti R., a cura di. International Marketing and the Country of Origin Effect: The Global Impact of "Made in Italy". Cheltenham: Edward Elgar Publishing.

Pucci T., Simoni C. e Zanni L. (2013). Modelli di business e strategie di marketing nelle medie imprese. La gestione della crisi tra continuità e cambiamento. Mercati e competitività, 3: 25-45, doi: 10.3280/MC2013-003003.

Resciniti R. e Matarazzo M. (2012). I nuovi mercati e le strategie di entrata delle medie imprese: evidenze e miti. Sinergie rivista di studi e ricerche, 89: 209230.

Tee R. e Gawer A. (2009) Industry Architecture as a Determinant of Successful Platform Strategies: A Case Study of the i-mode Mobile Internet Service. European Management Review (6)4: 217-232, doi: 10.1057/emr.2009.22. 